\title{
Ipsilesional trajectory control is related to contralesional arm paralysis after left hemisphere damage
}

\author{
Kathleen Y. Haaland $\cdot$ Sydney Y. Schaefer $\cdot$ \\ Robert T. Knight · John Adair · Alvaro Magalhaes • \\ Joseph Sadek · Robert L. Sainburg
}

Received: 17 September 2008 / Accepted: 29 April 2009 / Published online: 29 May 2009

(c) The Author(s) 2009. This article is published with open access at Springerlink.com

\begin{abstract}
We have recently shown ipsilateral dynamic deficits in trajectory control are present in left hemisphere damaged (LHD) patients with paresis, as evidenced by impaired modulation of torque amplitude as response amplitude increases. The purpose of the current study is to determine if these ipsilateral deficits are more common with contralateral hemiparesis and greater damage to the motor system, as evidenced by structural imaging. Three groups of right-handed subjects (healthy controls, LHD stroke patients with and without upper extremity paresis) performed single-joint elbow movements of varying ampli-
\end{abstract}

K. Y. Haaland ( $\varangle)$

Research Service (151),

New Mexico Veterans Affairs Healthcare System,

1501 San Pedro SE, Albuquerque, NM 87108, USA

e-mail: khaaland@unm.edu

K. Y. Haaland · J. Adair

Department of Neurology, University of New Mexico,

Albuquerque, NM, USA

K. Y. Haaland · J. Sadek

Department of Psychiatry, University of New Mexico,

Albuquerque, NM, USA

S. Y. Schaefer

Department of Biomedical Engineering,

Washington University, St. Louis, MO, USA

e-mail: syschaefer@gmail.com

S. Y. Schaefer

Program in Physical Therapy,

Washington University, St. Louis, MO, USA

R. T. Knight

Department of Psychology, University of California,

Berkeley, CA, USA

e-mail: rtknight@Berkely.edu tudes with their left arm in the left hemispace. Only the paretic group demonstrated dynamic deficits characterized by decreased modulation of peak torque (reflected by peak acceleration changes) as response amplitude increased. These results could not be attributed to lesion volume or peak velocity as neither variable differed across the groups. However, the paretic group had damage to a larger number of areas within the motor system than the non-paretic group suggesting that such damage increases the probability of ipsilesional deficits in dynamic control for modulating torque amplitude after left hemisphere damage.

\section{R. T. Knight}

Helen Wills Neuroimaging Center,

University of California, Berkeley, CA, USA

J. Adair

Neurology Service, New Mexico Veterans Affairs Healthcare

System, 1501 San Pedro SE, Albuquerque, NM 87108, USA

e-mail: john.adair@va.gov

\section{A. Magalhaes}

Radiology Service, New Mexico Veterans Affairs Healthcare System, 1501 San Pedro SE, Albuquerque, NM 87108, USA

e-mail: acamagalhaes@msn.com

\author{
A. Magalhaes \\ Department of Radiology, University of New Mexico, \\ Albuquerque, NM, USA \\ J. Sadek \\ Behavioral Healthcare Line (116), \\ New Mexico Veterans Affairs Healthcare System, \\ 1501 San Pedro SE, Albuquerque, NM 87108, USA \\ e-mail: jsadek@unm.edu \\ R. L. Sainburg \\ Department of Kinesiology, \\ The Pennsylvania State University, University Park, PA, USA
}


Keywords Paresis, lateralization - Motor control · Stroke $\cdot$ Ipsilateral impairment $\cdot$ Hemisphere

Motor cortex, premotor cortex, motor performance . Hemiparesis $\cdot$ Hemisphere, arm paralysis

\section{Introduction}

The contribution of the ipsilateral hemisphere to motor control has been demonstrated in humans through functional imaging studies in healthy adults (Kim et al. 1993; Kawashima et al. 2000; Haaland et al. 2004a) as well as by the ipsilesional deficits reported after left or right hemisphere damage (Wyke 1967; Fisk and Goodale 1988; Haaland and Harrington 1989; Winstein and Pohl 1995). Kinematic analysis of arm reaching has shown that the left hemisphere appears to be specialized for controlling initial trajectory features, including specification of initial joint torque (Fisk and Goodale 1988; Haaland and Harrington 1989; Haaland et al. 2004b; Winstein and Pohl 1995; Desrosiers et al. 1996). We recently showed that paretic patients with left hemisphere damage demonstrated ipsilesional deficits in dynamic control characterized by impaired ability to modulate torque amplitude (measured by peak acceleration) and enhanced ability to modulate torque duration (measured by acceleration duration) as movement amplitude increased. However, none of these studies have assessed whether these ipsilesional deficits are dependent on damage to the motor system significant enough to produce paresis; some have examined only paretic patients (Winstein and Pohl 1995; Schaefer et al. 2007) while others have included paretic and non-paretic patients (Fisk and Goodale 1988; Haaland et al. 2004b). None have directly compared the performance of paretic and non-paretic patients in order to assess the influence of significant damage to the motor system on these findings.

There is considerable evidence in animal and human studies that the motor cortex is important for controlling

\footnotetext{
R. L. Sainburg

Department of Neuroscience,

The Pennsylvania State University,

University Park, PA, USA

R. L. Sainburg

Department of Bioengineering,

The Pennsylvania State University,

University Park, PA, USA

R. L. Sainburg

The Gerontology Center,

The Pennsylvania State University,

University Park, PA, USA

e-mail: rls4s@psu.edu
}

many of the dynamic aspects of contralateral movement, such as velocity and force (Kurtzer et al. 2005; Richardson et al. 2006; Turner et al. 1998) and specification of initial trajectory features (Drew et al. 2008). These findings suggest that the ipsilesional deficits seen after left hemisphere damage may be influenced by greater damage to the motor system, especially motor cortex. Therefore, the purpose of the current study is to determine if our previously reported dynamic deficits in left hemisphere damaged (LHD) stroke patients with hemiparesis (Schaefer et al. 2007) are present only in hemiparetic patients with greater damage to the motor system. More specifically, for single-joint movements of different amplitude, we predict reduced scaling of joint torque amplitude in paretic patients with LHD and greater damage to the motor system, relative to healthy control subjects and LHD patients without paresis. In addition, if modulation of torque duration is utilized to compensate for errors in modulation of torque amplitude, as has been suggested (Mutha and Sainburg 2007; Fisher et al. 2000), the paretic group should demonstrate greater enhancement of torque duration modulation relative to the other two groups.

\section{Materials and methods}

\section{Participants}

Twenty-two right-handed stroke patients with LHD (10 with upper extremity paresis and 12 without paresis) and 24 right-handed healthy control subjects were examined after obtaining approval from the Human Research and Review Committee of the University of New Mexico School of Medicine and informed consent from each participant, according to the Declaration of Helsinki. Eight of the control subjects and five of the paretic subjects were included in a previous study (Schaefer et al. 2007). All subjects were screened and excluded based on history of substance abuse, serious psychiatric diagnosis, non-stroke neurological diseases for the stroke groups and all neurological diagnoses for the control group, or peripheral movement restrictions, such as neuropathy or orthopedic disorders. All raw grip strength measures were converted to standardized T-scores (Mean $=50, \mathrm{SD}=10$ ) based on published normative data (Heaton et al. 2004). Upper extremity paresis was defined by a right, contralesional grip strength 1.5 standard deviations below the published normative standards and at least 1.5 standard deviations less than standardized left, ipsilesional grip strength using a hand dynamometer. All stroke patients completed the experiment with their ipsilesional, left arm. The healthy control group, which was age- and education-matched with the two stroke groups, also performed the movement task with their left hand. 
Table 1 Demographic and descriptive data

\begin{tabular}{|c|c|c|c|}
\hline Variable $($ mean $\pm \mathrm{SD})$ & Control & Non-paretic & Paretic \\
\hline$n$ & 24 & 12 & 10 \\
\hline Age (years) & $62.3 \pm 8.5$ & $65.0 \pm 9.4$ & $55.7 \pm 12.5$ \\
\hline Education (years) & $15.4 \pm 2.4$ & $16.0 \pm 2.9$ & $14.7 \pm 2.6$ \\
\hline Sex (\% male $)$ & 75.0 & 75.0 & 90.0 \\
\hline Years post-stroke & & $5.8 \pm 4.9$ & $7.2 \pm 5.8$ \\
\hline Lesion volume $\left(\mathrm{cm}^{3}\right)$ & & $56.7 \pm 58.8$ & $80.1 \pm 53.3$ \\
\hline Fugl-Meyer UE motor ${ }^{\mathrm{a}}$ & & $61.2 \pm 3.2$ & $32.7 \pm 20.1$ \\
\hline Auditory comprehension ${ }^{\mathrm{b}}$ & $79.5 \pm 1.8$ & $72.2 \pm 16.1$ & $71.3 \pm 10.7$ \\
\hline Grip strength right ${ }^{\mathrm{c}}$ & $48.8 \pm 7.7$ & $45.0 \pm 9.1$ & $10.6 \pm 12.2$ \\
\hline Grip strength left ${ }^{c}$ & $48.6 \pm 6.5$ & $52.9 \pm 10.0$ & $49.1 \pm 5.6$ \\
\hline
\end{tabular}

Mean $\pm \mathrm{SD}$, except for sex

${ }^{\text {a }}$ Maximum $=66$ (Fugl-Meyer et al. 1975)

b Western aphasia battery, maximum $=80$ (Kertesz 1982)

c $\mathrm{T}$-score based on published norms (Heaton et al. 2004)

Table 1 summarizes the characteristics of each group. There were no significant differences across all groups in age, education, sex, or ipsilesional grip strength. The two stroke groups showed no significant differences in time post-stroke or lesion volume. Contralesional Fugl-Meyer motor scores, another measure of upper extremity paresis (Fugl-Meyer et al. 1975; Woodbury et al. 2007), were more impaired in the paretic group than the non-paretic group $[F(1,20)=23.7, P<0.001]$. Group differences were present for contralesional grip strength $[F(2,43)=63.8, P<0.001]$ related to poorer grip in the paretic group relative to the non-paretic group $(P<0.001)$ and the control group $(P<0.001)$.

\section{Lesion size and location}

The MR examinations were performed on a $1.5 \mathrm{~T}$ system (Edge, Picker) in all of the non-paretic patients and in nine of the ten paretic patients. One patient had a CT scan (Picker). CT slice thickness was $8 \mathrm{~mm}$ with no gap between slices, and MRI slice thickness was $5 \mathrm{~mm}$. A board-certified neurologist (RK), who was blinded to the behavioral characteristics of the patients, outlined the area of damage for each patient on 11 standardized horizontal sections derived from the DeArmond atlas (DeArmond et al. 1989) using T1 weighted MRI images for anatomical detail and T2 weighted FLAIR images to specify borders of the damaged tissue. These tracings were retraced on a digitizing tablet for input into a computer program that used an algorithm to calculate lesion volume and location within each hemisphere (Frey et al. 1987). This information was used to ensure comparable lesion size and intrahemispheric location.
Experimental setup

The experimental setup is described in detail in a previous publication (Schaefer et al. 2007). Participants sat facing a projection screen with their left arm supported over a horizontal surface by an air-jet system to reduce the effects of friction and gravity. The start circle, a target, and a cursor that represented finger position were projected on a horizontal back-projection screen positioned above the arm, with a horizontal mirror positioned below this screen. The mirror reflected the visual display to give the illusion that the display was in the same horizontal plane as the fingertip.

All joints distal to the elbow were immobilized with an adjustable brace. Position and orientation of the segments proximal and distal to the elbow joint were sampled using a Flock of Birds (FoB) ${ }^{\circledR}$ (Ascension-Technology) magnetic six-degree-of-freedom (6-DOF) movement recording system. Sensors were attached to the upper arm segment and to the air sled where the forearm was fitted. Our custom software used the FoB sensor data to compute the three-dimensional (3D) position of the index finger tip and to project a cursor onto the screen that reflected fingertip position. Digital data were collected at $103 \mathrm{~Hz}$ and custom computer algorithms for experiment control and data analysis were written in REAL BASICTM (REAL Software Inc.), C and IgorPro $^{\mathrm{TM}}$ (Wavemetric Inc.).

\section{Experimental task}

The location of the circular targets $(2.5 \mathrm{~cm}$ diameter $)$ were determined according to subjects' shoulder and elbow angles, and were unique for each subject. For all subjects, the upper arm was positioned at $20^{\circ}$, and stabilized by a brace attached to the table. The starting elbow angle was $80^{\circ}$, while the targets were placed at the fingertip locations that required $15^{\circ}, 25^{\circ}$, and $45^{\circ}$ elbow extensions, respectively, or an average of 7,18 , or $30 \mathrm{~cm}$ movements, respectively. These target extents were similar to those in two previous studies (Sainburg and Schaefer 2004; Schaefer et al. 2007) and were chosen in order to systematically vary velocity and distance of the hand in the mid-range of elbow joint motion.

The cursor, which corresponded to the position of the index fingertip, the start circle, and the target were displayed on the screen prior to each trial. Subjects held the cursor within the start circle for 200 milliseconds to initiate each trial. They were instructed to move the finger to the target using a single, uncorrected motion in response to an audiovisual "go" signal. The finger cursor was removed at the "go" signal. For motivational purposes, subjects received a numerical score, which was based on final position accuracy. Movement speed was self selected, but all subjects received velocity feedback after each trial in the 
form of a progress-bar display that showed subjects the peak velocity achieved during the trial. This display also showed a targeted velocity range (between 0.6 and $2.0 \mathrm{~m} / \mathrm{s}$ ). If the subjects did not achieve a velocity within this range, they did not receive points based on accuracy. We employed this feedback in order to minimize velocity differences between the patient and the control groups. In addition, during the 45 practice trials they were given verbal feedback to move between 0.6 and $2.0 \mathrm{~m} / \mathrm{s}$. Targets were presented in a pseudorandom order, such that no single target was presented consecutively. The first 45 trials of each session allowed for task familiarity and were excluded from all analyses. Kinematic and statistical analyses were conducted on the following 150 trials. Trials were excluded if subjects triggered data acquisition by moving outside the start circle, but did not make a movement to the target.

\section{Kinematic data}

The 3D position of the index finger, elbow point, and shoulder point were calculated from sensor position and orientation data. Then, joint angles were calculated from these data. All kinematic data were low-pass filtered at $8 \mathrm{~Hz}$ (3rd order, dual-pass Butterworth), and differentiated to yield tangential velocity and acceleration values. Movement start was determined by identifying the time of peak velocity and searching backward in time for the first minimum below $6 \%$ of peak tangential velocity, or for zero velocity, whichever was identified first. Movement end was similarly determined by searching forward in time from peak velocity to find the first minimum below $6 \%$ of peak tangential velocity.

\section{Dependent measures}

Absolute final position error was calculated as the absolute value of the distance from the finger tip at movement end to the center of the target regardless of whether the movement overshot or undershot the target. Variable error, a measure of consistency for each subject, was calculated as the variabilities of the distance from the finger tip at movement end to the mean final position for each target. Movement time was defined as the elapsed time from movement start to movement end. Peak velocity was defined as the absolute maximum tangential velocity. Peak acceleration was defined as the absolute maximum tangential acceleration and peak deceleration was defined as the absolute minimum tangential acceleration. These measures were normalized relative to the amplitude of such signals within each subject to reduce between-subject variability while preserving within-subject trends of modulation. Thus, in this study, peak acceleration and deceleration were normalized to percent of largest peak acceleration and peak deceleration, respectively, within each subject across targets. These normalized values (\% max) were calculated for each trial $i$ for each subject: (1) $\mathrm{acc}_{\text {normi }}=\left[\left(\mathrm{acc}_{\max }-\mathrm{acc}_{i}\right) / \mathrm{acc}_{\max }\right] \times 100$ where $\mathrm{acc}_{\max }=$ largest peak acceleration produced by that subject during the session and $\mathrm{acc}_{i}=$ peak acceleration for trial $i$; (2) $\operatorname{dec}_{\text {normi }}=\left[\left(\left|\operatorname{dec}_{\max }\right|-\left|\operatorname{dec}_{i}\right|\right) /\left|\operatorname{dec}_{\max }\right|\right] \times 100$ where $\operatorname{dec}_{\max }=$ largest (most negative) peak deceleration produced by that subject during the session and $\operatorname{dec}_{i}=$ peak deceleration for trial $i$. Acceleration duration was defined as the elapsed time from movement start to time of peak velocity, and deceleration duration was defined as the elapsed time from time of peak velocity to movement end. Since there is a direct relationship between tangential (hand) acceleration and joint (elbow) torque during singlejoint movements, our measures of acceleration amplitude and duration reflect the amplitude and duration of muscle forces acting at the elbow joint.

\section{Statistical analysis}

Our task was designed to randomly vary the required amplitude of movements between the shortest $\left(15^{\circ}\right)$ and longest $\left(45^{\circ}\right)$ target distance, which served as our independent variable for this study. Both of these targets were within the mid-range of elbow joint motion. We interposed an intermediate length target $\left(25^{\circ}\right)$ in order to randomize target amplitudes. Thus, we limited our statistical analysis to the short and long target distances, which corresponded to substantial changes in our independent variables.

The individual dependent measures were analyzed using two-way mixed model analysis of variance (ANOVA), with group (control, paretic, non-paretic) as the between-subject factor, and target extent $\left(15^{\circ}\right.$ and $\left.45^{\circ}\right)$ as the within-subject factor. Significant two-way interactions were followed with three pairwise group $\times$ target ANOVAs comparing the control group with each of the stroke groups and the stroke groups with each other. When significant group differences were present, Tukey post hoc tests were performed to identify the source of the group differences. Our major hypotheses were that the paretic group would demonstrate less change in peak acceleration as response amplitude increased, reflecting impaired scaling of torque amplitude and enhanced modulation of acceleration duration as response amplitude increased, reflecting enhanced scaling of torque duration. Therefore, we predicted a significant group $\times$ target interaction for these two measures.

\section{Results}

Task performance

Group $\times$ target ANOVAs identified significant main effects of target for all dependent measures $(P<0.01$ with large 
Table 2 Performance for short $\left(15^{\circ}\right)$ and long $\left(45^{\circ}\right)$ movements

\begin{tabular}{|c|c|c|c|c|c|c|}
\hline \multirow{2}{*}{$\begin{array}{l}\text { Groups } \\
\text { Targets }\end{array}$} & \multicolumn{2}{|l|}{ Control } & \multicolumn{2}{|l|}{ Non-paretic } & \multicolumn{2}{|l|}{ Paretic } \\
\hline & $15^{\circ}$ & $45^{\circ}$ & $15^{\circ}$ & $45^{\circ}$ & $15^{\circ}$ & $45^{\circ}$ \\
\hline MT (ms) & $715.8(35)$ & 987.7 (37) & $773.7(50)$ & $1,056.3(52)$ & $927.7(54)$ & $1,243.0(57)$ \\
\hline $\mathrm{AE}(\mathrm{cm})$ & $1.5(0.2)$ & $1.8(0.1)$ & $1.6(0.3)$ & $2.2(0.2)$ & $1.4(0.3)$ & $2.3(0.2)$ \\
\hline VE (cm) & $1.1(0.2)$ & $1.4(0.1)$ & $1.1(0.2)$ & $1.4(0.1)$ & $0.9(0.3)$ & $1.6(0.1)$ \\
\hline PeakDec (\% max) & $24.8(1.3)$ & $61.6(1.6)$ & $22.2(1.8)$ & $56.6(2.3)$ & $24.8(1.9)$ & $60.0(2.5)$ \\
\hline DecDur (ms) & 424.7 (21) & $563.0(26)$ & $461.6(30)$ & $587.5(37)$ & $469.3(33)$ & $634.6(41)$ \\
\hline
\end{tabular}

Mean (SEM)

$M T$ movement time, $A E$ absolute error, $V E$ variable error, PeakDec normalized peak deceleration, DecDur deceleration duration

effect sizes, $\eta^{2}>0.18$, in all cases) in the expected directions (e.g., increased peak velocity as amplitude increased). These effects are well established (Brown and Cooke 1981; Cooke and Brown 1990) and not the focus of this study. Rather, because our hypotheses predicted that paresis would be associated with differences in the way that peak acceleration and acceleration duration change with target amplitude, we focused on group $\times$ target interactions.

As can be seen in Table 2, ipsilesional movement time differed across the three groups $[F(2,43)=7.1, P<0.05]$ due to systematically longer MTs for the paretic group relative to the control $(P<0.001)$ and the non-paretic $(P=0.052)$ groups with no significant differences between the control and non-paretic group $(P=0.526)$. Thus, only the paretic group showed prolonged movement durations in the ipsilesional arm relative to both other groups though the finding was marginal when comparing the two stroke groups. The group $\times$ target interaction was not significant for MT $(P=0.651)$. In contrast, and as predicted, there were no significant group or group $\times$ target interaction effects for absolute or variable error, peak deceleration or deceleration duration $(P$ values ranged from 0.243 to 0.977), as seen in Table 2 .

\section{Velocity and acceleration}

Previous studies have consistently reported a strong tendency for subjects to scale movement speed with movement distance when reaching to different target distances (Brown and Cooke 1981; Cooke and Brown 1990; Schaefer et al. 2005).

Figure 1a displays such distance scaling of the velocity profiles of representative subjects from each group. Distance scaling of velocity (Fig. 2c) is present in all three groups to a similar degree, as evidenced by a significant main effect of target $[F(1,43)=668.4, P<0.001]$ and the lack of a significant group $\times$ target interaction $(P=0.14)$. In addition, despite the appearance of group differences in Fig. 1, there was no main effect of group $(P=0.15)$ These findings suggest that the group $\times$ target interactions in our other dependent measures cannot be accounted for by such interactions in peak velocity.

However, there were mechanistic differences in how each group scaled velocity with target extent. Figure $1 \mathrm{~b}$ displays the acceleration profiles of representative subjects from each group, and shows the peak amplitude of acceleration increased substantially with target distance for the control and non-paretic patient, but not the paretic stroke patient. In contrast, the paretic stroke patient's acceleration duration (marked by where the acceleration pulse crosses zero) increased with target distance producing effective scaling of peak tangential velocity with intended movement distance. Yet, the non-paretic patient showed normal modulation of peak acceleration and only marginally greater changes in acceleration duration.

These differences were consistent across subjects for peak acceleration (Fig. 1c). The ipsilesional arm of the paretic group showed less of an increase in peak acceleration as distance increased relative to that of the non-paretic and control groups. This is reflected by a significant group (control, non-paretic, paretic) $\times$ target interaction $[F(2,43)=$ $4.9, P<0.05]$ and significant group $\times$ target interactions only when the paretic group was included in the two-group ANOVAs [paretic and non-paretic $F(1,20)=4.4, P=0.05$; paretic and control $F(1,32)=8.1, P<0.01$; non-paretic and control $F(1,34)=0.5, P=0.48]$.

Figure 1c shows a more marginal pattern of results for acceleration duration: Greater target-dependent change in acceleration duration was present in the paretic group as compared to the control group. However, the non-paretic group showed marginally greater target-dependent increases in acceleration duration relative to the control group and lesser target-dependent increases relative to the paretic group. These relationships were reflected by a significant interaction of group and target when all three groups were compared $[F(2,43)=6.1, P<0.01]$ with greater increases in acceleration duration as target increased for the paretic group relative to the control group $[F(1,32)=14.3, P=0.001]$, but no such group $\times$ target interaction effects when the non-paretic group was compared 
Healthy control

a

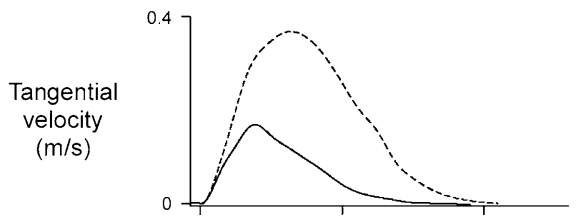

b

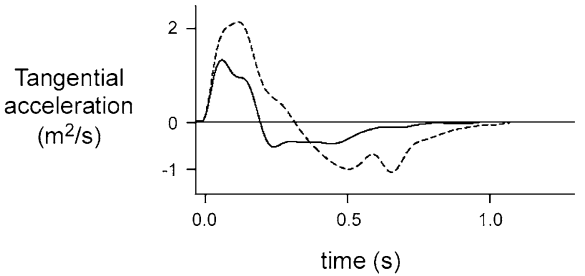

Non-paretic stroke

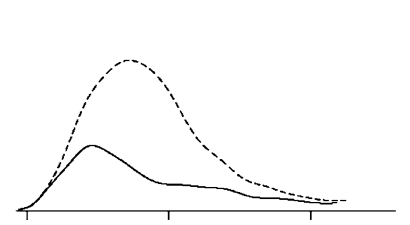

Paretic stroke

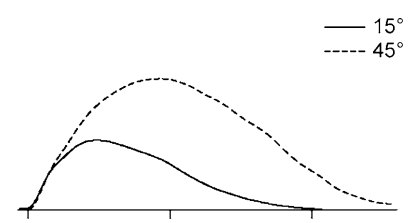

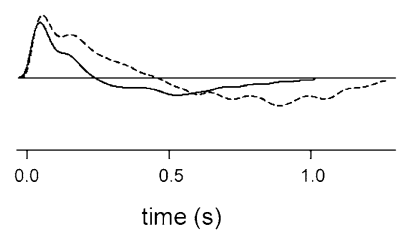

C
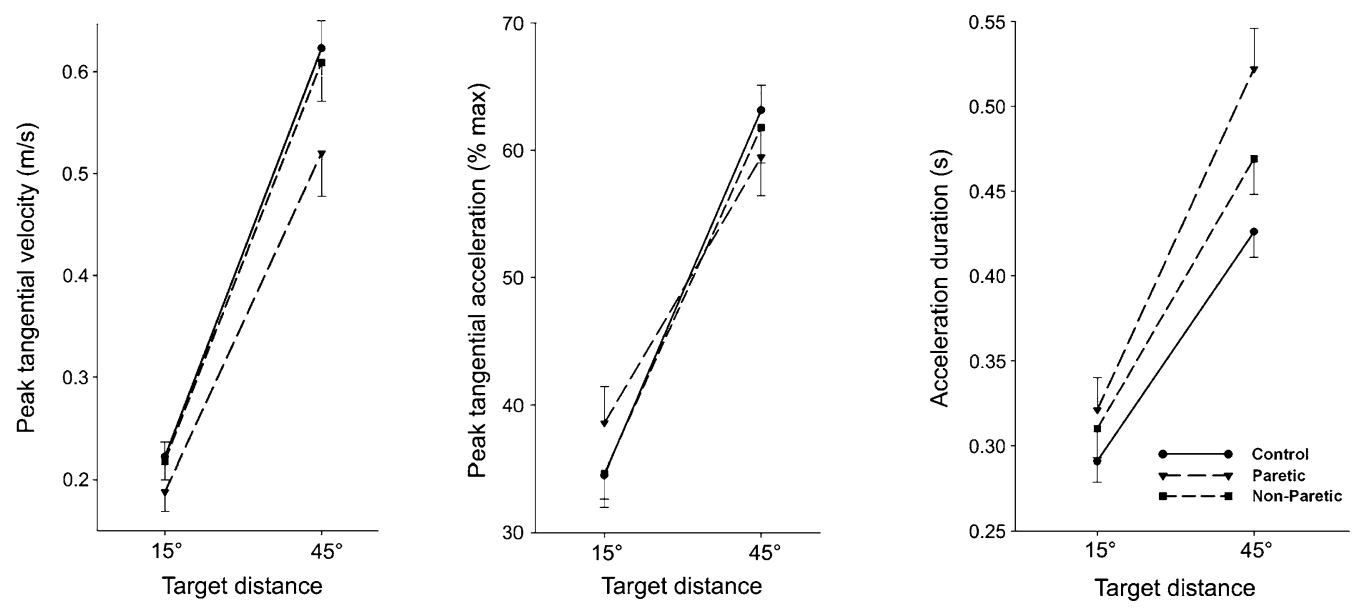

Fig. 1 a Average tangential velocity profiles and $\mathbf{b}$ average tangential acceleration profiles for each target for a representative subject from each group. c Mean peak velocity, peak acceleration (normalized to \%

max) and acceleration duration for each target is displayed for the three groups. Bars indicate standard error of the mean

to the control group $\left[F(1,34)=2.2, P=0.14, \eta^{2}=0.06\right.$ (small effect size)] or to the paretic group $[F(1,20)=2.4$, $P=0.14, \eta^{2}=0.11$ (medium effect size)]. Thus, enhancement in scaling of acceleration duration across targets did not differentiate the paretic and non-paretic groups in a convincing way.

\section{Neuroanatomical correlates}

Figure 2 displays the lesion overlap for the two stroke groups. There was greater overlap in the paretic than the non-paretic group, especially in the insula, basal ganglia and internal capsule. When damage was tabulated for different areas more of the paretic patients $(100 \%)$ than the non-paretic patients $(67 \%)$ had damage in at least one part of the motor system (somatomotor cortex, internal capsule), and this incidence rate did not change if basal ganglia and insula involvement were included. When the incidence of damage to less than two motor regions or two or more regions was compared, the paretic group had a higher incidence of the latter $(90 \%)$ than the non-paretic group (33\%)
(Fisher Exact Test, $P=0.011$ ). This discrepancy was due more to greater subcortical (e.g., internal capsule, basal ganglia) involvement in the paretic $(80 \%)$ than the nonparetic $(33 \%)$ groups than to differences in somatomotor cortex involvement in the two groups (paretic 60\%, nonparetic $67 \%$ ). Finally, on slice 9, Fig. 2, there was evidence of greater incidence of damage in the centrum semiovale/ corona radiata in the paretic $(60-79 \%)$ than the non-paretic $(0-19 \%)$ group. The figure suggests that none of the nonparetic patients had damage in one part of this centrum semiovale region. Based on the Schmahmann and Pandya (2006) monkey atlas, such damage appears to interrupt interhemispheric callosal projections, intrahemispheric connections between premotor regions and other parts of somatomotor cortex, and internal capsule.

\section{Discussion}

This study extends our previous findings, suggesting that the left hemisphere may be specialized for specification of 
a

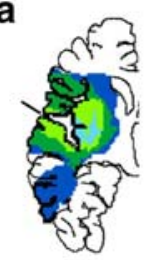

4

b

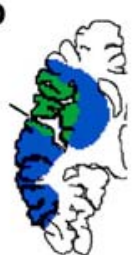

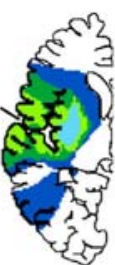

5

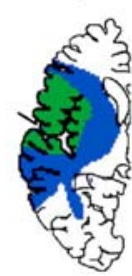

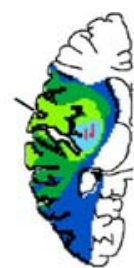

6

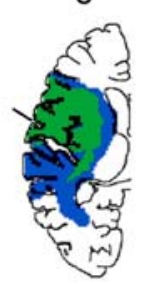

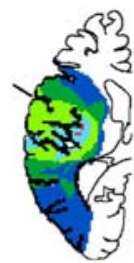

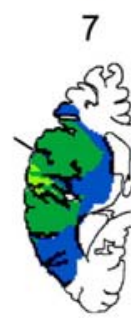

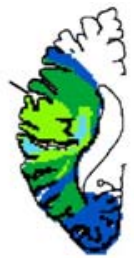

8

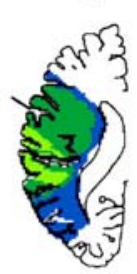

Fig. 2 Lesion locations were based upon superimposing lesion tracings from MRI or CT images on axial slices for left hemisphere damaged stroke patients $\mathbf{a}$ with upper extremity paresis and $\mathbf{b}$ without upper
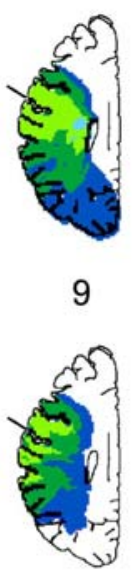

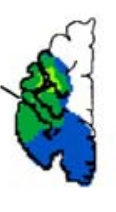

10

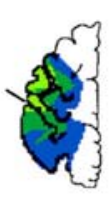

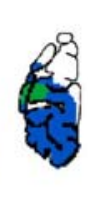

11

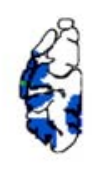

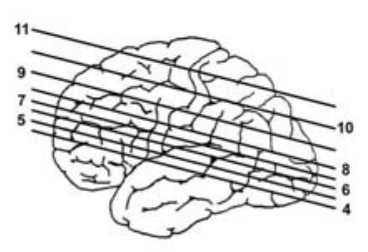

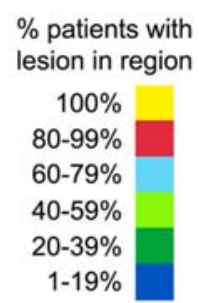

extremity paresis. Colors of shaded regions denote percentage (20, 40, 60,80 , or $100 \%$ ) of patients with lesion in the corresponding area. Tic marks the central sulcus

distances that is different in the paretic group. It has previously been well established that the amplitude of singlejoint movements is normally controlled by two independent mechanisms that separately specify the magnitude and duration of the initial phase of the joint torque profile (Gordon et al. 1995; Gordon and Ghez 1987a, b). Both of these mechanisms combine to determine the amplitude of peak movement velocity. When subjects make movements of different distances from a start position, they tend to scale peak velocity with movement distance. This scaling occurs by the scaling of the initial peak torque amplitude, a direct result of scaling peak agonist activity. In addition to this, the duration of the initial torque profile also scales with peak velocity, and has been shown to correspond to the duration of agonist muscle activity and the onset time of antagonist muscle activity (Bermejo and Zeigler 1989; Gordon and Ghez 1987a, b). Whereas the scaling of initial torque amplitude reflects movement planning, the scaling of torque duration is also substantially influenced by sensory feedback during the course of the movement (Brown and Cooke 1981). Our previous findings in unilaterally damaged stroke patients with hemiparesis indicated that these two processes were differentially affected, depending on the hemisphere that had been lesioned (Schaefer et al. 2007). These results were consistent with our studies in neurologically intact young right-handers, which showed that movements of the right arm achieve different velocities and distances primarily by scaling of peak torque amplitude, with little modulation of torque duration. In contrast, movements of the left hand were initiated with a stereotyped torque amplitude, and peak velocity was scaled by modulation of torque duration (Sainburg and Schaefer 2004). Based on these findings, we hypothesized that each hemisphere might have become differentially specialized 
for each of these processes, a finding that was supported by our previous findings in right and left hemisphere damaged stroke patients (Schaefer et al. 2007).

Whether or not these differences in strategy reflect a "choice" by the central nervous system is not clear. However, the idea that one can voluntarily modify the shape of an acceleration profile seems unlikely, and certainly has not been demonstrated previously. We would like to stress that nervous system damage might very well modify the strategy that individuals typically employ in order to carry out a task. However, we also expect that flexibility in the central nervous system is unlikely to be completely eliminated, such that other "choices" of strategy probably could be developed with training. We, thus assert that understanding the strategic "choices" that subject groups systematically employ cannot only provide information about the function of hemispheric contributions to movement, but could provide important information for rehabilitation intervention. Overall, it is important to emphasize that the differences that we are stressing are not simply moving slower, or faster, but rather in changing the acceleration or torque profiles, associated with scaling of speed across distances.

In the current paper, we tested the hypothesis that the dominant arm/left hemisphere specialization for specification of torque amplitude should depend on the severity of contralesional motor deficit induced by LHD. This hypothesis is based on the idea that motor circuits in the left hemisphere are recruited for specifying torque amplitude (and presumably other dynamic factors) for the left, as well as right arm. However, because motor function and deficits are a multifaceted phenomenon, and because out patient groups were restricted in size, we did not attempt to represent motor function as a continuous variable. Rather, we identified patients with clear clinical hemiparesis, and those without hemiparesis to provide two groups that reflected two distinct levels of motor deficits, patients with and without identifiable contralesional hemiparesis.

Our current findings showed that ipsilesional acceleration (or torque) amplitude modulation was differentially affected in LHD patients with and without contralesional arm paresis. More specifically, patients with contralesional upper extremity paresis showed the most substantial restrictions in modulation of acceleration amplitude in the ipsilesional upper extremity. These findings were confirmed by a significant group by target interaction for peak acceleration. Even when acceleration amplitude remains constant across movement distances, torque duration can be modulated to vary the amplitude of peak velocity. In fact, modulation of torque duration has been shown to play a "compensatory" role, correcting for errors in initial planning of torque amplitude (Bermejo and Zeigler 1989; Fisher et al. 2000). Initial undershooting in torque amplitude can be compensated during the evolving movement by extending torque duration. Thus, we believe the paretic group's greater increase in ipsilesional torque duration modulation is a compensatory response that reflects the influence of the intact right hemisphere, which we have previously suggested is better adapted for modulating torque duration (Schaefer et al. 2007). In addition, this enhancement of right hemisphere influence could also be related to reduced inhibition of the right hemisphere due to left hemisphere damage; it has previously been shown that damage to one hemisphere reduces inhibition of the undamaged hemisphere when compared to inhibition induced by transcranial magnetic stimulation (TMS) of motor cortex in neurologically intact subjects (Kobayashi et al. 2003).

Therefore, we speculate that in the paretic group enhanced modulation of torque duration is related to compensation for errors in torque amplitude scaling as well as decreased inhibition of right hemisphere functioning. The more marginal increase in torque duration scaling in the non-paretic group likely reflects a reduction in interhemispheric inhibition of the right hemisphere only related to left hemisphere damage rather than compensation. This conclusion is based upon the non-paretic group's normal modulation of torque amplitude. Therefore, there were no errors in the initial phase of the movement that required correction. In addition, the similar degree of motor cortex damage in the paretic and non-paretic groups would also predict comparable decreases in right hemisphere inhibition because decreases in interhemispheric inhibitory effects have been linked to motor cortex damage only (Kobayashi et al. 2003). It should be emphasized that these speculations require direct confirmation using TMS in paretic and nonparetic LHD stroke patients.

There are many potential neural mechanisms that could explain these ipsilesional findings, given the number of cortical areas that directly connect to the corticospinal tract (Dum and Strick 1991) and the influence of the basal ganglia and cerebellum (Middleton and Strick 2000) on movement. Two potential circuits for ipsilateral control of movement have received the greatest attention, and the primary disagreement between these two theories is the output pathway, not the cortical or subcortical regions that are critical for controlling the central processing, which in this case is pulse height control.

The first theory emphasizes that ipsilateral motor control is subserved by the same cortical regions and output pathways that subserve contralateral motor control. This view capitalizes on the fact that even though a very small portion of the corticospinal tract projects to the ipsilateral spinal cord (Brinkman and Kuypers 1973; Jane et al. 1967; Glees and Cole 1952; Porter and Lemon 1993), these projections affect ipsilateral movement. This view is also consistent with the involvement of corticobulbar pathways, which have stronger ipsilateral connections than the corticospinal 
system (Brodal 1973), but suffers from the fact that direct ipsilateral control is quite small.

The second explanation relates ipsilateral motor control of torque amplitude modulation to the same cortical regions that subserve control for contralateral movements except that information is transmitted through the corpus callosum to motor output pathways in the opposite hemisphere. Callosal apraxia, which is characterized by limb apraxia in the left, but not the right arm, is a good example of this explanation. The left hemisphere is specialized for limb praxis (Ochipa and Rothi 2000), and callosal apraxia is attributed to callosal damage, which prevents the intact spatiotemporal representations purportedly localized in the left hemisphere from influencing gestures of the left arm only. In contrast, these representations are available to control the right, contralateral arm directly through the contralateral efferent motor pathways (Geschwind 1965). Therefore, despite intact ipsilesional projections in the left corticospinal tract, limb apraxia was still present in the left arm. This argues against the importance of the ipsilateral corticospinal and corticobulbar fibers for explaining the ipsilesional apraxic deficits. Such explanations may also be important for the ipsilesional deficits in torque amplitude scaling that we have reported. Our finding of greater incidence of damage in the centrum semiovale/corona radiata of the hemiparetic group who also demonstrate deficits in scaling torque amplitude is consistent with the potential importance of callosal pathways. However, the location of the damage also may affect the fibers in the internal capsule, which would argue that the efferent pathways projecting from the left hemisphere also influence the ipsilesional pulse height deficits.

\section{Clinical implications}

These results demonstrate that contralesional, right hemiparesis and greater damage to the motor system is associated with dynamic control deficits after left hemisphere stroke Because hemiparetic stroke patients use their ipsilesional arm five to six times more frequently than their contralesional arm alone (Vega-Gonzalez and Granat 2005), such deficits in the ipsilesional limb may have significant functional implications. The relationship between deficits in the modulation of torque amplitude and deficits in real world activities has not been examined. However, it has been shown that the hand path curvature of multi-joint movements in different directions, another dynamic characteristic of the movement trajectory, is associated with functional deficits (Schaefer et al. 2006). In that study, we showed that less efficient performance on simulated activities of living (Jebsen Hand Function Test) were related to greater trajectory curvature or less direct and efficient movements when performing reaching movements.
Acknowledgments This research was supported by a Department of Veterans Affairs Medical Merit Review and Rehabilitation Merit Review (B4476), the National Institutes of Health, National Institute for Child Health and Human Development (\#RO1HD39311), National Institute on Aging training grant, Interdisciplinary Training in Gerontology (\#T32AG00048). We gratefully acknowledge (1) Venkat Mannam for lesion analysis, (2) Jennifer Hogan, Rena Singleton, Lee Stapp, and Jennifer Rinehart for data collection, and (3) Dr. Sally Harris, HealthSouth Rehabilitation Hospital, Lovelace Medical Center, and Lovelace Rehabilitation Hospital for patient referral.

Open Access This article is distributed under the terms of the Creative Commons Attribution Noncommercial License which permits any noncommercial use, distribution, and reproduction in any medium, provided the original author(s) and source are credited.

\section{References}

Bagesteiro LB, Sainburg RL (2003) Nondominant arm advantages in load compensation during rapid elbow joint movements. J Neurophysiol 90(3):1503-1513

Bermejo R, Zeigler HP (1989) Prehension in the pigeon. II. Kinematic analysis. Exp Brain Res 75:577-585

Brinkman J, Kuypers HG (1973) Cerebral control of contralateral and ipsilateral arm, hand and finger movements in the split-brain rhesus monkey. Brain 96:653-674

Brodal A (1973) Self-observations and neuro-anatomical considerations after a stroke. Brain 96:675-694

Brown SH, Cooke JD (1981) Responses to force perturbations preceding voluntary human arm movements. Brain Res 220:350-355

Cooke JD, Brown SH (1990) Movement-related phasic muscle activation. II. Generation and functional role of the triphasic pattern. J Neurophysiol 63:465-472

DeArmond SJ, Fusco MM, Dewey MM (1989) Structure of the human brain: a photographic atlas. Oxford University Press, New York

Desrosiers J, Bourbonnais D, Bravo G, Roy PM, Guay M (1996) Performance of the 'unaffected' upper extremity of elderly stroke patients. Stroke 27:1564-1570

Drew T, Andujar JE, Lajoie K, Yakovenko S (2008) Cortical mechanisms involved in visuomotor coordination during precision walking. Brain Res Rev 57:199-211

Duff SV, Sainburg RL (2007) Lateralization of motor adaptation reveals independence in control of trajectory and steady-state position. Exp Brain Res 179:551-561

Dum RP, Strick PL (1991) Premotor areas: nodal points for parallel efferent systems involved in the central control of movement. In: Humphrey DR, Freund H-J (eds) Motor control: concepts and issues. Wiley, New York, pp 383-397

Fisher BE, Winstein CJ, Velicki MR (2000) Deficits in compensatory trajectory adjustments after unilateral sensorimotor stroke. Exp Brain Res 132:328-344

Fisk JD, Goodale MA (1988) The effects of unilateral brain damage on visually guided reaching: hemispheric differences in the nature of the deficit. Exp Brain Res 72:425-435

Frey RT, Woods DL, Knight RT, Scabini D, Clayworth C (1987) Defining functional areas with averaged CT scans. Soc Neurosci Abstr 13:1266

Fugl-Meyer AR, Jaasko L, Leyman I, Olsson S, Steglind S (1975) The post-stroke hemiplegic patient. 1. A method for evaluation of physical performance. Scand J Rehabil Med 7:13-31

Geschwind N (1965) Disconnexion syndromes in animals and man. Brain 88:237-294

Glees P, Cole J (1952) Ipsilateral representation in the cerebral cortex; its significance in relation to motor function. Lancet 1:1191-1192 
Gordon J, Ghez C (1987a) Trajectory control in targeted force impulses. II. Pulse height control. Exp Brain Res 67:241-252

Gordon J, Ghez C (1987b) Trajectory control in targeted force impulses. III. Compensatory adjustments for initial errors. Exp Brain Res 67:253-269

Gordon J, Ghilardi MF, Ghez C (1995) Impairments of reaching movements in patients without proprioception. I. Spatial errors. J Neurophysiol 73:347-360

Haaland KY, Harrington DL (1989) Hemispheric control of the initial and corrective components of aiming movements. Neuropsychologia 27:961-969

Haaland KY, Elsinger CL, Mayer AR, Durgerian S, Rao SM (2004a) Motor sequence complexity and performing hand produce differential patterns of hemispheric lateralization. J Cogn Neurosci 16:621-636

Haaland KY, Prestopnik JL, Knight RT, Lee RR (2004b) Hemispheric asymmetries for kinematic and positional aspects of reaching. Brain 127:1145-1158

Heaton RK, Miller SW, Taylor MJ, Grant I (2004) Revised comprehensive norms for an expanded Halstead Reitan battery: demographically-adjusted neuropsychological norms for African American and Caucasian adults. Psychological Assessment Resources, Lutz, FL

Jane JA, Yashon D, DeMyer W, Bucy PC (1967) The contribution of the precentral gyrus to the pyramidal tract of man. J Neurosurg 26:244-248

Kawashima R, Okuda J, Umetsu A, Sugiura M, Inoue K, Suzuki K, Tabuchi M, Tsukiura T, Narayan SL, Nagasaka T, Yanagawa I, Fujii T, Takahashi S, Fukuda H, Yamadori A (2000) Human cerebellum plays an important role in memory-timed finger movement: an fMRI study. J Neurophysiol 83:1079-1087

Kertesz A (1982) Western aphasia battery. The Psychological Corporation, New York

Kim SG, Ashe J, Hendrich K, Ellermann JM, Merkle H, Ugurbil K, Georgopoulos AP (1993) Functional magnetic resonance imaging of motor cortex: hemispheric asymmetry and handedness. Science 261:615-617

Kobayashi M, Hutchinson S, Schlaug G, Pascual-Leone A (2003) Ipsilateral motor cortex activation on functional magnetic resonance imaging during unilateral hand movements is related to interhemispheric interactions. Neuroimage 20:2259-2270

Kurtzer I, Herter TM, Scott SH (2005) Random change in cortical load representation suggests distinct control of posture and movement. Nat Neurosci 8:498-504
Middleton FA, Strick PL (2000) Basal ganglia and cerebellar loops: motor and cognitive circuits. Brain Res Rev 31:236-250

Mutha PK, Sainburg RL (2007) Control of velocity and position in single joint movements. Hum Mov Sci 26:808-823

Ochipa C, Rothi LJG (2000) Limb apraxia. Semin Neurol 20:471-478

Porter R, Lemon R (1993) Corticospinal function and voluntary movement. Clarendon Press, Oxford

Richardson AG, Overduin SA, Valero-Cabre A, Padoa-Schioppa C, Pascual-Leone A, Bizzi E, Press DZ (2006) Disruption of primary motor cortex before learning impairs memory of movement dynamics. J Neurosci 26:12466-12470

Sainburg RL, Schaefer SY (2004) Interlimb differences in control of movement extent. J Neurophysiol 92:1374-1383

Schaefer SY, Sainburg R, Haaland KY (2005) Differential hemispheric contributions to unilateral arm movements. Society of Neuroscience Abstracts

Schaefer SY, Haaland KY, Sainburg R (2006) Left but not right hemisphere damage produces ipsilesional deficits in intersegmental coordination. Society of Neuroscience Abstracts 181

Schaefer SY, Haaland KY, Sainburg RL (2007) Ipsilesional motor deficits following stroke reflect hemispheric specializations for movement control. Brain 130:2146-2158

Schmahmann JD, Pandya DN (2006) Fiber pathways of the brain. Oxford University Press, New York

Turner RS, Grafton ST, Votaw JR, DeLong MR, Hoffman JM (1998) Motor subcircuits mediating the control of movement velocity: a PET study. J Neurophysiol 80:2162-2176

Vega-Gonzalez A, Granat MH (2005) Continuous monitoring of upper-limb activity in a free-living environment. Arch Phys Med Rehabil 86:541-548

Winstein CJ, Pohl PS (1995) Effects of unilateral brain damage on the control of goal-directed hand movements. Exp Brain Res 105:163-174

Woodbury ML, Velozo CA, Richards LG, Duncan PW, Studenski S, Lai SM (2007) Dimensionality and construct validity of the Fugl-Meyer assessment of the upper extremity. Arch Phys Med Rehabil 88:715-723

Wyke M (1967) Effect of brain lesions on the rapidity of arm movement. Neurology 17:1113-1120 